\title{
HOMOGENEOUS INVERSE LIMIT SPACES WITH NONREGULAR COVERING MAPS AS BONDING MAPS
}

\author{
JAMES T. ROGERS, JR. AND JEFFREY L. TOLLEFSON
}

Abstract. We construct counterexamples to the conjecture that, in an inverse sequence $(X, f)$ of closed manifolds $X_{n}$ with covering maps $f_{m}^{n}: X_{n} \rightarrow X_{m}$ as bonding maps, if the inverse limit space is homogeneous, then there exists an integer $m$ such that (for all $n>m$ ) the covering map $f_{m}^{n}: X_{n} \rightarrow X_{m}$ is regular.

1. Introduction. In $1966 \mathrm{R}$. M. Schori [5] conjectured that, in an inverse limit sequence $(X, f)$ of closed manifolds $X_{n}$ with covering maps $f_{m}^{n}: X_{n} \rightarrow X_{m}$ as bonding maps, if the inverse limit space is homogeneous, then there exists an integer $m$ such that (for all $n>m$ ) the covering $\operatorname{map} f_{m}^{n}: X_{n} \rightarrow X_{m}$ is regular. We present counterexamples to this conjecture in all dimensions greater than one.

This conjecture is the converse to McCord's theorem [2] that if $(X, f)$ is an inverse limit sequence of closed manifolds and each bonding map $f_{1}^{n}: X_{n} \rightarrow X_{1}$ is a regular covering map, then the limit space is homogeneous. Examples of inverse limit sequences of closed manifolds with covering maps as bonding maps where the limit spaces fail to be homogeneous have been given in [3] and [5].

2. Preliminaries and notation. We let $(X, f)$ denote the inverse limit sequence with factor spaces $X_{n}$ and bonding maps $f_{m}^{n}: X_{n} \rightarrow X_{m}$ $(n \geqq m \geqq 1)$. The limit space, $\lim (X, f)$, is denoted by $X_{\infty}$. If each factor space $X_{n}$ is a closed, connected manifold and each bonding map $f_{m}^{n}$ a covering map, then we refer to $(X, f)$ as a weak solenoidal sequence and to $X_{\infty}$ as a weak solenoidal space. We follow the notation in [1] for inverse limits. The reader is referred to [4] for the theory of covering spaces.

Lemma 1. Let $A, B$, and $C$ be manifolds and let $f: A \rightarrow B$ and $g: B \rightarrow C$ be covering maps. If the composite $g \circ f: A \rightarrow C$ is a regular covering map, then $f$ is also a regular covering map.

We assume throughout that basepoints have been chosen nicely and are fixed.

\section{LEMMA 2. In the commutative diagram}

Received by the editors October 19, 1970.

AMS 1969 subject classifications. Primary 5425; Secondary 5480.

Key words and phrases. Inverse limits, weak solenoidal spaces, normal series, covering map, homogeneity. 


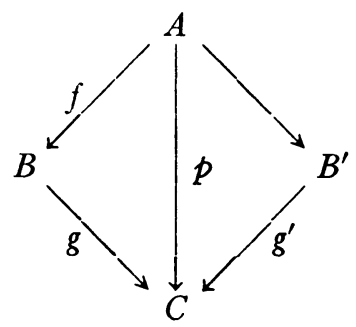

of manifolds and finite-to-one regular covering maps, let $p_{*} \pi_{1}(A)$ $=\left(g_{*} \pi_{1}(B)\right) \cap\left(g_{*}^{\prime} \pi_{1}\left(B^{\prime}\right)\right)$. Moreover, suppose the multiplicity $\alpha$ of $g$ is relatively prime to the multiplicity $\alpha^{\prime}$ of $g^{\prime}$. Then $g^{\prime}$ has the property that it can be factored into the composition of the covering maps $B^{\prime} \stackrel{h}{\rightarrow} D \stackrel{h^{\prime}}{\rightarrow} C$ such that $h^{\prime}$ is not regular if and only if $f$ also has this property.

Proof. The factorization of $g^{\prime}$ into $h^{\prime} \circ h$ is equivalent to the existence of a nonnormal subgroup of $\pi_{1}(C)$ that contains $g_{*}^{\prime} \pi_{1}\left(B^{\prime}\right)$, namely $h_{*}^{\prime} \pi_{1}(D)$. It is sufficient to observe that $\pi_{1}(B) / f_{*} \pi_{1}(A)$ is isomorphic to $\pi_{1}(C) / g_{*}^{\prime} \pi_{1}\left(B^{\prime}\right)$, since this implies that a nonnormal subgroup containing $f_{*} \pi_{1}(A)$ occurs in $\pi_{1}(B)$ if and only if one containing $g_{*}^{\prime} \pi_{1}\left(B^{\prime}\right)$ occurs in $\pi_{1}(C)$. But the indices $\alpha$ and $\alpha^{\prime}$ of $g_{*} \pi_{1}(B)$ and $g_{*}^{\prime} \pi_{1}\left(B^{\prime}\right)$, respectively, in $\pi_{1}(C)$ are relatively prime and so $\pi_{1}(C)$ is the smallest subgroup of itself containing both. Hence the above isomorphism follows from the isomorphism theorem.

3. Equivalent sequences. Let $(X, f)$ be a weak solenoidal sequence in which each bonding map $f_{1}^{n}: X_{n} \rightarrow X_{1}$ is a regular covering map. The limit space $X_{\infty}$ is homogeneous [2]. Let us assume that each map $f_{n}^{n+1}: X_{n+1} \rightarrow X_{n}$ can be factored as the composition of a regular covering map $\lambda_{n}: X_{n+1} \rightarrow Y_{n}$ and a nonregular covering map $h_{n}: Y_{n} \rightarrow X_{n}$. We have the commutative diagram

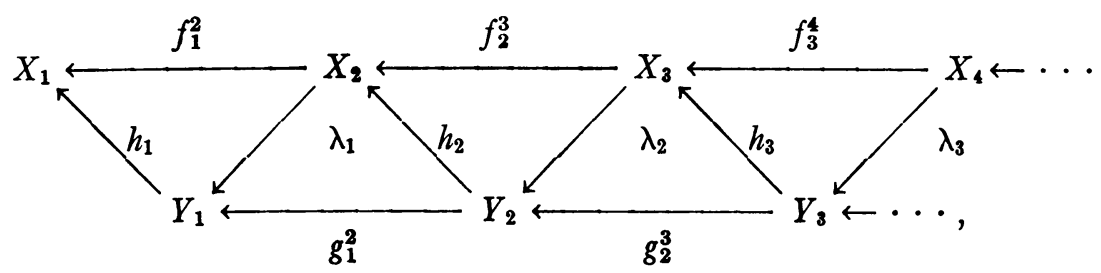

where we define $g_{n}^{n+1}=\lambda_{n} \circ h_{n+1}$. The bottom row determines a weak solenoidal sequence $(Y, g)$ with the property that no bonding map $g_{m}^{n}(n>m)$ is regular (see Lemma 1). However, $Y_{\infty}=\lim (Y, g)$ is clearly homeomorphic to $X_{\infty}$ and is thus homogeneous.

In the next section we construct weak solenoidal sequences $(X, f)$ that can be factored in this manner. 
4. Construction of $(X, f)$. Let $G$ be the group with presentation $\left(a, b: a^{\alpha}=1, b^{\alpha^{2}}=1, a^{-1} b a=b^{\alpha+1}\right)$, where $\alpha$ is a positive prime integer. $G_{\alpha}$ is a nonhamiltonian group of order $\alpha^{3}$. Let $X_{1}$ be a closed $n$ manifold such that there is an epimorphism $\eta_{\alpha}: \pi_{1}\left(X_{1}\right) \rightarrow G_{\alpha}$ for every prime $\alpha$. For example, we could let $X_{1}$ be the $n$-manifold $(n \geqq 2)$ obtained from two triangulated copies of $S^{1} \times S^{n-1}$ by removing the interior of a polyhedral $n$-cell from each and then matching the resulting $(n-1)$-sphere boundaries by a piecewise linear homeomorphism (this is the connected sum, denoted by $\left.\left(S^{1} \times S^{n-1}\right) \#\left(S^{1} \times S^{n-1}\right)\right)$. Then $\pi_{1}\left(X_{1}\right) \cong Z_{*} Z$ if $n>2$, and $\pi_{1}\left(X_{1}\right) \cong(a, b, c, d:[a, b][c, d]=1)$ if $n=2$.

Let $\alpha_{2}<\alpha_{3}<\ldots$ be a fixed sequence of prime integers. For each $i \geqq 2$ define $p_{i}: A_{i} \rightarrow X_{1}$ to be the covering map such that $\left(p_{i}\right)_{*} \pi_{1}\left(A_{i}\right)$ $=$ the kernel of $\eta_{\alpha_{i}}$. Then $p_{i}$ is a regular covering map of multiplicity $\alpha_{i}^{3}$ and factors into the composition of a regular and a nonregular covering map (since $\pi_{1}\left(X_{1}\right) /\left(p_{i}\right)_{*} \pi_{1}\left(A_{i}\right) \cong G_{\alpha_{i}}$ has a nonnormal subgroup). Since $p_{i}$ has a finite multiplicity, $A_{i}$ is a closed manifold.

We define the sequence $(X, f)$ inductively. Suppose then that $X_{1}$ has already been fixed as described in the preceding paragraph. Define $X_{2}=A_{2}$ and $f_{1}^{2}: X_{2} \rightarrow X_{1}$ to be $p_{2}$. Now suppose we have defined $X_{i}$ and $f_{i-1}^{i}: X_{i} \rightarrow X_{i-1}$ (for $i \leqq k$ ) such that each $f_{1}^{i}: X_{i} \rightarrow X_{1}$ is a regular covering map of multiplicity $\alpha_{2} \alpha_{3} \cdots \alpha_{i}$ and each $f_{i-1}^{i}$ factors into a regular and a nonregular covering map. To define $f_{k}^{k+1}: X_{k+1} \rightarrow X_{k}$ consider the covering maps $f_{1}^{k}: X_{k} \rightarrow X_{1}$ and $p_{k+1}: A_{k+1} \rightarrow X_{1}$. Define $f_{1}^{k+1}: X_{k+1} \rightarrow X_{1}$ to be the covering map such that

$$
\left(f_{1}^{k+1}\right)_{*} \pi_{1}\left(X_{k+1}\right)=\left[\left(f_{1}^{k}\right)_{*} \pi_{1}\left(X_{k}\right)\right] \cap\left[\left(p_{k+1}\right)_{*} \pi_{1}\left(A_{k+1}\right)\right] .
$$

$f_{1}^{k+1}$ can be factored through $f_{1}^{k}$, so define $f_{k}^{k+1}$ such that $f_{1}^{k+1}=f_{1}^{k} \circ f_{k}^{k+1}$. Clearly $f_{1}^{k+1}$ is a regular covering map. Furthermore, by Lemma 2, the regular covering map $f_{k}^{k+1}$ can be factored into a regular and a nonregular covering map since $p_{k+1}$ has this property. This defines the weak solenoidal sequence $(X, f)$ that produces the desired counterexample.

5. Questions. Let $(X, f)$ and $(Y, g)$ be weak solenoidal sequences. We say that $(X, f)$ is equivalent to $(Y, g)$ if there is a third weak solenoidal sequence in which subsequences of both $(X, f)$ and $(Y, g)$ can be embedded. For example, the sequences $(X, f)$ and $(Y, g)$ of $\$ 3$ are equivalent.

QUESTION 1. If $X_{\infty}=\lim (X, f)$ is a homogeneous weak solenoidal space, is $(X, f)$ equivalent to another weak solenoidal sequence $(Y, g)$ in which each of the bonding maps $g_{1}^{n}$ are regular covering maps? 
QUESTION 2. Let $X_{\infty}$ be a weak solenoidal space in which all the path components are homeomorphic. Is $X_{\infty}$ homogeneous?

Acknowledgement. We are grateful to Professor L. Sonneborn for a helpful discussion.

\section{REFERENCES}

1. S. Eilenberg and N. Steenrod, Foundations of algebraic topology, Princeton Univ. Press, Princeton, N. J., 1952. MR 14, 398.

2. M. C. McCord, Inverse limit sequences with covering maps as bonding maps, Trans. Amer. Math. Soc. 114 (1965), 197-209. MR 30 \#3450.

3. J. T. Rogers, Jr. and J. L. Tollefson, Involutions on solenoidal spaces, Fund. Math. (to appear).

4. E. H. Spanier, Algebraic topology, McGraw-Hill, New York, 1966. MR 35 $\# 1007$.

5. R. M. Schori, Inverse limits and homogeneity, Trans. Amer. Math. Soc. 124 (1966), 533-539. MR 33 \#6574.

Tulane University, New Orleans, Louisiana 70118

Iowa State University, Ames, Iowa 50010 